\title{
AN EXPLICIT CONSTRUCTION OF CASIMIR OPERATORS AND EIGENVALUES : I
}

\author{
H. R. Karadayi and M. Gungormez \\ Dept.Physics, Fac. Science, Tech.Univ.Istanbul \\ 80626, Maslak, Istanbul, Turkey
}

\begin{abstract}
We give a general method to construct a complete set of linearly independent Casimir operators of a Lie algebra with rank N. For a Casimir operator of degree p, this will be provided by an explicit calculation of its symmetric coefficients $g^{A_{1} A_{2} . . A_{p}}$. It is seen that these coefficients can be described by some rational polinomials of rank $\mathrm{N}$. These polinomials are also multilinear in Cartan sub-algebra indices taking values from the set $I_{\circ} \equiv\{1,2, \ldots, N\}$. The crucial point here is that for each degree one needs, in general, more than one polinomial. This in fact is related with an observation that the whole set of symmetric coefficients $g^{A_{1} A_{2} . . A_{p}}$ is decomposed into some sub-sets which are in one-to-one correspondence with these polinomials. We call these sub-sets clusters and introduce some indicators with which we specify different clusters. These indicators determine all the clusters whatever the numerical values of coefficients $g^{A_{1} A_{2} . . A_{p}}$ are. For any degree p, the number of clusters is independent of rank N. This hence allows us to generalize our results to any value of rank $\mathrm{N}$.

To specify the general framework, explicit contructions of 4th and 5th order Casimir operators of $A_{N}$ Lie algebras are studied and all the polinomials which specify the numerical value of their coefficients are given explicitly.
\end{abstract}

e-mail: karadayi@sariyer.cc.itu.edu.tr 


\section{INTRODUCTION}

For a classical or exceptional Lie algebra $\mathbf{G}$, the problem of finding explicit expressions for Casimir operators and their eigenvalues is of principal importance both in physics and in mathematics. A Casimir operator $\mathbf{I}(\mathbf{p})$ of degree $\mathrm{p}$ can be expressed by

$$
I(p) \equiv \sum_{A_{1} \leq A_{2} \leq \ldots \leq A_{p}} g^{A_{1}, A_{2} . . A_{p}} \operatorname{sym}\left(T_{A_{1}} T_{A_{2}} . . T_{A_{p}}\right)
$$

where the sum is over indices taking values from the set

$$
S \equiv\{1,2, . . \operatorname{dim} \mathbf{G}\}
$$

The coefficients $g^{A_{1}, A_{2} . . A_{p}}$ can be assumed to be completely symmetric and hence $\mathbf{s y m}(\ldots)$ means complete symmetrization with weight 1 for the generators $T_{A}$ of $\mathbf{G}$. For a D-dimensional representation, corresponding eigenvalues can then be expressed by

$$
\frac{1}{\mathbf{D}} \operatorname{Trace}(\mathbf{I}(\mathbf{p}))
$$

The expression (I.1) is in principle due to Poincare-Birkhoff-Witt (PBW) theorem while (I.3) is a result of Schur lemma [1]. One must note here that (I.1) has an abstract meaning which is valid for any representation of $\mathbf{G}$ whereas (I.3) must be calculated for each representation separately. In physics litterature, there are several works [2] dealing with appropriate descriptions of these two expressions. We also emphasize the works of Okubo and Patera [3] concerning a study of fourth and fifth order Casimirs which we now consider as preliminaries to our work. Extensions to superalgebras are made in some relatively recent works [4] and there are recent efforts for generalizations to q-superalgebras [5]. For affine Kac-Moody algebras, an extension of the generalized second order Casimir element to higher orders has also been accomplished [6].

On the other hand, the results of all these efforts find applications in string theories. A spectacular example is the well-known anomaly cancellation mechanism [7] which is based on some very special non-linear relationships among eigenvalues (I.3) of operators (I.1). These were for the days of first string revolution. In the present days of second string revolution [8], it is seen that all Casimir invariants are involved in a highly non-trivial way . For instance, the relevant operators of $\mathrm{N}=2$ supersymmetric models [9] can be expressed in terms of Casimir operators.

Beside applications, it is known [2] that a complete knowledge for a Lie algebra can be obtained with a complete knowledge for all its independent Casimir operators and their eigenvalues. Hence, for all irreducible representations, an actual calculation of (I.3) is a worthwhile task in principle as well as in practical applications. To this end, one of the essential problems is to calculate the multiplicity of weights participating in representations and this problem is also at the root of Weyl character formulas or recursive multiplicity formulas which are due to Freudenthal and Kostant. Explicit calculation of traces could still be complicated for irreducible matrix representations having higher dimensions. This will be considered in the second part of our work which is based on the mechanism presented in a previous article [10].

Another problem appears in the determination of the relationships which are known to exist among different Casimir operators of the same Lie algebra. This is indeed a natural problem when one considers that Casimir elements are generally defined on the universal enveloping algebra with a PBW basis. As is known [1] a PBW basis is formed by monomials constructed from multiple products of Lie algebra generators. It is therefore natural to ask some relationships among different Casimir elements if one wants only a finite number of them. One can think such relationships in two ways:

(i) Non-linear dependences among Casimir elements of different orders,

(ii) Linear dependences among Casimir elements of the same order.

Due to illuminating works of Borel and Chevalley [11] and possibly some others working on group cohomologies, the problem (i) has been solved with the calculation of Betti numbers of topological spaces formed by group manifolds. These Betti numbers are known, in the same time, to be exponents which specify the degrees of Racah invariants [12]. The Racah invariants also provide us a way to determine the Casimir operators which are independent non-linearly. Gruber and O'Raifertaigh [13] show us a generalization of 
these invariants. It is however well-known that all these constructions are not unique, i.e. there could always be some other choices and clearly this has to do with (ii). If one recalls, on the contrary of our choice in (I.1), that Casimir elements are in general non-homogeneous superpositions of PBW monomials, it is seen that this last problem has also some basic features. In two successive works, we will give a unified picture for these problems. For $\mathbf{A}_{\mathbf{N}}$ Lie algebras, a general solution to (i) is as in the following

Let $\kappa(\mathbf{p})$ be the number of linearly independent Casimir operators expressed in the homogeneous form of (I.1). Then,

$$
\kappa(p)=\text { the number of partitions of } p \text { into all positive integers except } 1 \text {. }
$$

Some examples will be instructive here:

(i) $\quad \kappa(4)=2 \quad$ due to $\quad 4=2+2$

(ii) $\quad \kappa(5)=2$ due to $5=3+2$

(iii) $\kappa(6)=4$ due to $6=4+2=3+3=2+2+2$

(iv) $\kappa(7)=4$ due to $7=5+2=4+3=3+2+2$

Our solutions for the numerical values of coefficients $g^{A_{1}, A_{2} . . A_{p}}$ have the generic form for $\mathrm{p}=4,5$

$$
P \text { parameter(1) }+Q \text { parameter }(2) \text {. }
$$

As will be shown in the following sections, the choice of two free parameters in (I.4) will be made as being in line with the partitions $4=2+2$ or $5=3+2$. The coefficients $\mathrm{P}$ and $\mathrm{Q}$ are rational polinomials of rank $\mathrm{N}$ and they are also multi-linear in Cartan sub-algebra indices $i_{1}, i_{2}, . . i_{p}$ taking values from the set $\mathbf{I}_{\circ} \equiv\{1,2, \ldots N\}$. It is crucial to note here that we always need several $\mathbf{P}$ and $\mathbf{Q}$ polinomials in order to describe the whole set of coefficients $\mathbf{g}^{\mathbf{A}_{1}, \mathbf{A}_{2} \ldots \mathbf{A}_{\mathbf{p}}}$. To this end, we introduce two novel concepts: clusters and indicators. The clusters are defined to be subsets of coefficients $g^{A_{1}, A_{2} . . A_{p}}$ with the same numerical value and the whole set of these coefficients has a direct sum decomposition in terms of these clusters. It is seen that a unique polinomial $\mathrm{P}$ or $\mathrm{Q}$ can be assigned only to a cluster and hence we need in general more than one polinomial for the whole set of coefficients. The indicators, on the other hand, are defined in such a way that they take different values on different clusters and hence they count the number of different clusters. The procedure which is introduced by the clusters and indicators works in an independent way from the rank $\mathrm{N}$ and this allows us to extend our results for all values of the rank $\mathrm{N}$.

Within the scope of this work, we expose only the results for $p=4,5$. The generalizations beyond $p=7$ begin to be difficult because the number of indicators increases and at present we can not be able to find a systematic procedure to determine all the indicators completely.

In section II, we will give some useful notation and introduce some generalized scalar products with which we define indicators. Our results for degrees $\mathrm{p}=4,5$ will be given respectively in sections (III) and (IV). Some features will also be emphasized in the last section.

\section{CLUSTERS AND INDICATORS}

Let us begin with a detailed description of the generator basis $T_{A}$ for a Lie algebra $\mathbf{G}$ ( $\equiv \mathbf{A}_{\mathbf{N}}$ ) which is defined by

$$
\left[T_{A}, T_{B}\right]=F_{A B}^{C} T_{C}
$$

$F_{A B}^{C}$ 's here are structure constants and indices A,B,C take values from the set $\mathrm{S}$, as in (I.2). We assume it has a triangular decomposition $S \equiv S_{+} \oplus S_{-} \oplus S_{\circ}$ in such a way that:

(i) $S_{+} \equiv\left\{1,2, . . \frac{1}{2} N(N+1)\right\}$

(ii) $S_{-} \equiv \frac{1}{2} N(N+1) \oplus S_{+}$

(iii) $S_{\circ} \equiv N(N+1) \oplus I_{\circ}$

For $i \in I_{\circ}$, the generators which correspond to simple roots $\alpha_{i}$ can be chosen by

$$
e_{\alpha_{i}} \equiv T_{i} \quad, \quad f_{\alpha_{i}} \equiv T_{i+\frac{1}{2} N(N+1)} \quad, \quad h_{\alpha_{i}} \equiv\left[e_{\alpha_{i}}, f_{\alpha_{i}}\right]=T_{i+N(N+1)} .
$$


and explicit matrix representations are always exist so that the set $\left\{e_{\alpha_{i}}, f_{\alpha_{i}}, h_{\alpha_{i}}\right\}$ forms a Chevalley basis. For the whole set of generators, one has a similar triangular decomposition $G \equiv G_{+} \oplus G_{-} \oplus G_{\circ}$ where $G_{\circ}$ is a Cartan sub-algebra and $G_{+} \oplus G_{\circ}$ one of its Borel sub-algebras. For this and other relevant techniques of Lie algebras, we will refer the excellent book of Humphreys [14]. For any irreducible representation specified by a dominant weight $\Lambda$, trace operations can be fixed by

$$
\operatorname{Trace}\left(T_{A} T_{B}\right)=c_{2}(\Lambda) g_{A B}
$$

where $g_{A B}$ is Killing-Cartan metric having the explicit matrix form $g=\operatorname{diagonal}(X, K)$. Here $\mathrm{X}$ is a $N(N+1) \times N(N+1) \quad$ dimensional symmetric sub-matrix with non-zero elements $X_{a, a+N(N+1) / 2}=1$ for $a \in S_{+} \oplus S_{-}$and $\mathrm{K}$ is just the Cartan matrix. $c_{2}(\Lambda)$ here is sometimes called the second index and it is normalized by

$$
c_{2}\left(\lambda_{i}\right)=\operatorname{Binomial}(N-1, i-1)
$$

for elementary representations of $A_{N}$ Lie algebras. Elementary representations are defined to be the ones characterized by fundamental dominant weights $\lambda_{i}$ which are duals of simple roots $\alpha_{i}$. They consist of only one Weyl orbit and hence exhibit no complications due to multiplicity problems encountered in their explicit matrix constructions. The numerical values of structure constants $F_{A B}^{C}$ are also fixed by

$$
F_{A C}^{D} F_{B D}^{C} \equiv 2(N+1) g_{A B}
$$

Now and then, summation is adopted over the repeated indices. All these completely determine the normalizations for which we use in the explicit construction of matrix representations.

The starting point now is

$$
F_{A B}^{\left\{C_{1}\right.} g^{\left.C_{2} \ldots C_{p-1}\right\} B}=0
$$

which is the result of invariance property of Casimir operators. Two-indexed solutions of (II.6) is just the inverse $g_{A B}^{-1} \equiv g^{A B}$ of Killing-Cartan metric. The following properties of coefficients $g^{A_{1}, A_{2}, . . A_{p}}$ would be quite helpful before solving (II.6) explicitly for $p \geq 4$ :

Invariance $: g^{A_{1}, A_{2}, . . A_{p} \text { 's }}$ are non-zero only for

$$
\alpha_{A_{1}}+\alpha_{A_{2}}+\ldots+\alpha_{A_{p}}=0
$$

where $\alpha_{I} \equiv 0$ for $I \in S_{\circ}$,

$\underline{\text { Parity }}: g^{A_{1}, A_{2}, . . A_{p}} \equiv g^{P\left(A_{p}\right), P\left(A_{p-1}\right), . . P\left(A_{1}\right)} \quad$ where $\quad \alpha_{P(A)} \equiv-\alpha_{A}$,

Duality : $g^{A_{1}, A_{2}, . . A_{p}} \equiv(-1)^{\sigma} g^{A_{1}{ }^{\star}, A_{2}{ }^{\star}, . . A_{p}{ }^{\star}} \quad$ where $\alpha_{A^{\star}}$ is just the conjugate of $\alpha_{A}$ under diagram automorphism of $A_{N}$ Lie algebras and $\sigma$ is a real phase which will be specified later,

Weyl Symmetry : Let us consider the action of Weyl group W: $\Theta\left(\alpha_{a}\right) \equiv \alpha_{\theta(a)}$ for all $\Theta \in W$. Note that $\bar{\Theta}$ is also an automorphism of subset $S_{+} \oplus S_{-}$. This will give us the possibility to extend Weyl reflections over the coefficients $g^{a_{1}, a_{2}, . . a_{p}}$ as in the following natural way:

$$
\Theta\left(g^{a_{1}, a_{2}, . . a_{p}}\right) \equiv g^{\theta\left(a_{1}\right), \theta\left(a_{2}\right), \ldots \theta\left(a_{p}\right)} \quad, \quad a_{1}, a_{2}, \ldots a_{p} \in S_{+} \oplus S_{-}
$$

It could be useful to explain all the notation here with an example. For this, let us consider, say, $A_{5}$ Lie algebra. As is defined above, the generator indices take values from the sets

(i) $S_{+} \equiv\{1,2, . .15\}$ for positive non-zero roots ,

(ii) $S_{-} \equiv\{16,17, . .30\}$ for negative non-zero roots ,

(iii) $S_{\circ} \equiv\{31,32, . .35\}$ for zero-roots . 
One has a lexicographical ordering for the composite roots in terms of simple roots $\alpha_{i} \quad(\mathrm{i}=1, \ldots 5)$ :

$$
\begin{aligned}
\alpha_{6} & =\alpha_{1}+\alpha_{2}, \alpha_{7}=\alpha_{2}+\alpha_{3}, \alpha_{8}=\alpha_{3}+\alpha_{4}, \alpha_{9}=\alpha_{4}+\alpha_{5}, \\
\alpha_{10} & =\alpha_{1}+\alpha_{2}+\alpha_{3}, \alpha_{11}=\alpha_{2}+\alpha_{3}+\alpha_{4}, \alpha_{12}=\alpha_{3}+\alpha_{4}+\alpha_{5}, \\
\alpha_{13} & =\alpha_{1}+\alpha_{2}+\alpha_{3}+\alpha_{4}, \alpha_{14}=\alpha_{2}+\alpha_{3}+\alpha_{4}+\alpha_{5}, \\
\alpha_{15} & =\alpha_{1}+\alpha_{2}+\alpha_{3}+\alpha_{4}+\alpha_{5}
\end{aligned}
$$

It will be instructive to show here that how invariance, parity, duality and Weyl-symmetry properties reduce the number of coefficients before solving them from (II.6). It is clear, for instance, that $g^{1,2,3,25}$ fulfills the invariance property while its equivalents are

$$
\begin{aligned}
& g^{1,2,3,25} \sim g^{10,16,17,18}, \\
& g^{1,2,3,25} \sim g^{3,4,5,27}
\end{aligned}
$$

due respectively to parity and duality properties. It has also several equivalents under the actions of Weylsymmetry. With respect to simple roots of $A_{5}$, its equivalents will be respectively

$$
\begin{aligned}
g^{1,2,3,25} & \sim g^{3,6,16,26} \\
g^{1,2,3,25} & \sim g^{6,7,17,25} \\
g^{1,2,3,25} & \sim g^{1,7,18,21} \\
g^{1,2,3,25} & \sim g^{1,2,8,28} \\
g^{1,2,3,25} & \sim g^{1,2,3,25}
\end{aligned}
$$

due to Weyl-symmetry.

All these properties restrict to some extent the number of coefficients $g^{A_{1}, A_{2}, \ldots A_{p}}$ which are unknowns of the equations (II.6) but it is readily seen that there are still a huge number of free parameters which simply made the generalizations difficult. It is therefore clear that the existence of clusters formed out of the coefficients $g^{A_{1}, A_{2}, \ldots A_{p}}$ having the same numerical value is of fundamental importance here and our main observation is that they can be determined by a set of properly chosen indicators. In order to define these indicators for degrees $\mathrm{p}=4,5$, we need two kinds of scalar products $\kappa_{1}$ and $\kappa_{2}$. First one of these is the usual one:

$$
\kappa_{1}(a, b) \equiv\left(\alpha_{a}, \alpha_{b}\right) \quad, \quad a, b \in S_{+} \oplus S_{-} .
$$

For root or weight lattices of classical and exceptional Lie algebras, such a scalar product are always defined on simple roots $\alpha_{i}$ by Cartan matrix elements $K_{i j} \equiv \frac{2\left(\alpha_{i}, \alpha_{j}\right)}{\left(\alpha_{j}, \alpha_{j}\right)}$. This can then be extended to the whole root or weight lattice when one recalls that roots are Z-linear and weights are Q-linear combinations of simple roots.

Our second scalar product is defined by

$$
\begin{array}{ll}
\kappa_{2}\left(i, \alpha_{j}+\ldots+\alpha_{j+k}\right)=n_{-}, & i<j \\
\kappa_{2}\left(i, \alpha_{j}+\ldots+\alpha_{j+k}\right)=n_{\circ}, & j \leq i \leq j+k \\
\kappa_{2}\left(i, \alpha_{j}+\ldots+\alpha_{j+k}\right)=n_{+}, & i>j+k .
\end{array}
$$

$n_{+}, n_{\circ}, n_{-}$here represent three different numbers. A choice $n_{-}=-1, n_{\circ}=2, n_{+}=1$ will be made in following chapters.

\section{FOURTH ORDER SOLUTIONS}

In chapters (III) and (IV), we assume $a_{1}, a_{2}, \ldots \in S_{+} \oplus S_{-}, I_{1}, I_{2}, \ldots \in S_{\circ}$ and $i_{1}, i_{2}, \ldots \in I_{\circ}$. It is now useful to study the whole set of coefficients $g^{A_{1}, A_{2} . . A_{p}}$ in the following four sub-classes:

$$
\begin{array}{ll}
\left(T^{(0)}\right) & g^{a_{1}, a_{2}, a_{3}, a_{4}}, \\
\left(T^{(1)}\right) & g^{a_{1}, a_{2}, a_{3}, I_{1}}, \\
\left(T^{(2)}\right) & g^{a_{1}, a_{2}, I_{1}, I_{2}}, \\
\left(T^{(4)}\right) & g^{I_{1}, I_{2}, I_{3}, I_{4}} .
\end{array}
$$


It would be helpful to recall here that indices $a_{1}, a_{2}, .$. are for non-zero roots while $I_{1}, I_{2}, .$. correspond to zero roots. Such a classification could therefore be considered to be suitable because, as we emphasized above, for the numerical values of coefficients $g^{A_{1}, A_{2} . . A_{p}}$ we expect some polinomials which are rational in $\mathrm{N}$ and also multi-linear in indices coming from the zero roots only. Within such a framework, it is natural to expect one polinomial for each one of the sub-classes in (III.1). This is trivial for $T^{(4)}$ but the more will be seen below for the other sub-classes. It will be seen that each sub-class $T^{(s)}$ is a direct sum of their clusters and each cluster is represented by a different polinomial. To specify the clusters, following definition of indicators seem to be the most convenient ones:

$$
\begin{aligned}
& \operatorname{IND}\left(T^{(0)}\right) \equiv \Sigma_{0}\left(\kappa_{1}\left(a_{1}, a_{2}\right), \kappa_{1}\left(a_{1}, a_{3}\right), \kappa_{1}\left(a_{1}, a_{4}\right), \kappa_{1}\left(a_{2}, a_{3}\right), \kappa_{1}\left(a_{2}, a_{4}\right), \kappa_{1}\left(a_{3}, a_{4}\right)\right) \\
& \operatorname{IND}\left(T^{(1)}\right) \equiv \Sigma_{1}\left(\kappa_{2}\left(I_{1}, \alpha_{a_{1}}\right), \kappa_{2}\left(I_{1}, \alpha_{a_{2}}\right), \kappa_{2}\left(I_{1}, \alpha_{a_{3}}\right)\right) \\
& \operatorname{IND}\left(T^{(2)}\right) \equiv \Sigma_{2}\left(\Gamma\left(\kappa_{2}\left(I_{1}, \alpha_{a_{1}}\right), \kappa_{2}\left(I_{1}, \alpha_{a_{2}}\right)\right), \Gamma\left(\kappa_{2}\left(I_{2}, \alpha_{a_{1}}\right), \kappa_{2}\left(I_{2}, \alpha_{a_{2}}\right)\right)\right)
\end{aligned}
$$

Let us first study the action of these indicators on $T^{(0)}$. It is sufficient to make this in the $A_{5}$ example given above because the results are independent of rank N. A set of appropriately chosen representatives is now

$$
g^{1,1,16,16}, g^{1,2,3,25}, g^{1,2,16,17}, g^{1,3,16,18} \in T^{(0)}
$$

on which the indicators act as

$$
\begin{aligned}
& \operatorname{IND}\left(g^{1,1,16,16}\right)=\Sigma_{0}(-2,-2,-2,-2, \quad 2, \quad 2) \equiv \Sigma_{0}(1) \\
& \operatorname{IND}\left(g^{1,2,3,25}\right)=\Sigma_{0}(-1,-1,-1,-1, \quad 0,0) \equiv \Sigma_{0}(2) \\
& \operatorname{IND}\left(g^{1,2,16,17}\right)=\Sigma_{0}(-2,-2,-1,-1, \quad 1,1) \equiv \Sigma_{0}(3) \\
& I N D\left(g^{1,3,16,18}\right)=\Sigma_{0}(-2,-2, \quad 0, \quad 0, \quad 0, \quad 0) \equiv \Sigma_{0}(4)
\end{aligned}
$$

The quantities $\Sigma$ are assumed to be completely symmetrical in their indices. Calculations can be made by the aid of simple fortran-programs for all other elements of $T^{0}$ and for any $A_{N}$ other than $A_{5}$. The results then show us that there are nothing else other than $\Sigma_{0}(k)$ 's for $\mathrm{k}=1,2,3,4$. We outline this fact by saying that the indicators receive four different values on the sub-class $T^{0}$. The following result reflects the relevance here:

For any $A_{N}$, any two elements $\mathrm{g}(1), \mathrm{g}(2) \in T^{0}$ have the same numerical value on condition that

$$
I N D(g(1))=I N D(g(2))
$$

This is the main observation which reveals us the existence of sub-sets which we would like to call clusters.

The similar analysis shows us that indicators take 4 and 6 different values on $T^{(1)}$ and $T^{(2)}$ respectively. To see this, it is sufficient to consider the representatives

$$
\begin{aligned}
& g^{1,2,21,31}, g^{1,2,21,32}, g^{2,3,22,31}, g^{1,2,21,33} \in T^{(1)} \\
& g^{2,17,31,31}, g^{2,17,31,32}, g^{2,17,31,33}, g^{1,16,31,31}, g^{1,16,32,32}, g^{1,16,31,32} \in T^{(2)}
\end{aligned}
$$

with corresponding actions

$$
\begin{aligned}
& \operatorname{IND}\left(g^{1,2,21,31}\right)=\Sigma_{1}(1, \quad 2,2) \equiv \Sigma_{1}(1) \\
& I N D\left(g^{1,2,21,32}\right)=\Sigma_{1}(-1,2,2) \equiv \Sigma_{1}(-1) \\
& I N D\left(g^{2,3,22,31}\right)=\Sigma_{1}(1,1,1) \equiv \Sigma_{1}(2) \\
& I N D\left(g^{1,2,21,33}\right)=\Sigma_{1}(-1,-1,-1) \equiv \Sigma_{1}(-2)
\end{aligned}
$$


and

$$
\begin{aligned}
& \operatorname{IND}\left(g^{2,17,31,31}\right)=\Sigma_{2}(1,1) \equiv \Sigma_{1}(1) \\
& I N D\left(g^{1,16,32,32}\right)=\Sigma_{2}(-1,-1) \equiv \Sigma_{1}(-1) \\
& \operatorname{IND}\left(g^{2,17,31,32}\right)=\Sigma_{2}(1,2) \equiv \Sigma_{1}(2) \\
& I N D\left(g^{1,16,31,32}\right)=\Sigma_{2}(-1,2) \equiv \Sigma_{1}(-2) \\
& I N D\left(g^{2,17,31,33}\right)=\Sigma_{2}(-1,1) \equiv \Sigma_{1}(3) \\
& I N D\left(g^{1,16,31,31}\right)=\Sigma_{2}(2,2) \equiv \Sigma_{1}(4) \text {. }
\end{aligned}
$$

It is seen here that we need to define an extra generator $\Gamma$ with the following values on sub-class $T^{(2)}$ :

$$
\begin{aligned}
& \Gamma(1,1) \equiv \Gamma(1), \\
& \Gamma(-1,-1) \equiv \Gamma(-1), \\
& \Gamma(2,2) \equiv \Gamma(2)
\end{aligned}
$$

As a result of this discussion, for any one of the coefficients $g^{A_{1}, A_{2}, A_{3}, A_{4}}$ of the fourth order Casimir we have one of the following polinomials:

$$
\begin{array}{ll}
g^{a_{1}, a_{2}, a_{3}, a_{4}} \equiv g_{4}(N) y_{k}(N), & k=1, . .4, \\
g^{a_{1}, a_{2}, a_{3}, I_{1}} \equiv g_{4}(N) y_{k}\left(i_{1}, N\right), & k=1,2, \\
g^{a_{1}, a_{2}, I_{1}, I_{2}} \equiv g_{4}(N) y_{k}\left(i_{1}, i_{2}, N\right), & k=1, . .4 \\
g^{I_{1}, I_{2}, I_{3}, I_{4}} \equiv g_{4}(N) y\left(i_{1}, i_{2}, i_{3}, i_{4}, N\right) &
\end{array}
$$

A point which is important especially for higher order Casimirs is the fact that the coefficients $g^{A_{1}, A_{2}, A_{3}, A_{4}}$ are in general rational polinomials of the rank N. It is therefore crucial to know here that

$$
g_{4}(N) \equiv \frac{1}{N(N-1)(N-2)} .
$$

Before attacking to solve (II.6), one must also recall that the number of coefficients with different numerical values is further reduced by the aid of the properties mentioned in section II. To this end, it is important to notice, in view of decompositions (III.9), that duality properties can be given most conveniently as in the following:

$$
\begin{aligned}
& y_{-k}\left(i_{1}, N\right)=(-1)^{1} y_{k}\left(N+1-i_{1}, N\right), k=1,2, \\
& y_{-k}\left(i_{1}, i_{2}, N\right)=(-1)^{2} y_{k}\left(N+1-i_{2}, N+1-i_{1}, N\right), k=1,4, \\
& y\left(i_{1}, i_{2}, i_{3}, i_{4}, N\right)=(-1)^{4} y\left(N+1-i_{4}, N+1-i_{3}, N+1-i_{2}, N+1-i_{1}, N\right) .
\end{aligned}
$$

All these have in mind, we can easily solve equations (II.6) for only a highly reduced number of unknown coefficients. This can be repeated for several values of rank $\mathrm{N}$ which make the following generalizations possible. For this, it will be useful to define some auxiliary polinomials $\rho_{r}(N),(r=0, . .4)$ with the following definitions:

$$
\begin{aligned}
& \rho_{0}(N)=(N-1) u(1)-N u(2) \\
& \rho_{1}(N)=2(N-1)(2 N-1) u(1)-3 N^{2} u(2) \\
& \rho_{2}(N)=-(N-1)\left(N^{2}-2 N+3\right) u(1)+N\left(N^{2}-N+1\right) u(2) \\
& \rho_{3}(N)=-2(N-1)\left(N^{2}+2\right) u(1)+N\left(2 N^{2}+N+2\right) u(2) \\
& \rho_{4}(N)=(N-1)(N-2)((2 N-10) u(1)-3 N u(2)) .
\end{aligned}
$$

The two free parameters $\mathrm{u}(1)$ and $\mathrm{u}(2)$ here are chosen with the following values of the unique polinomial representing elements of the subset $T^{(4)}$ :

$$
y(1,1,1,1, N) \equiv u(1) \quad, \quad y(1,1,2,2, N) \equiv u(2) .
$$


Note here that the choices (III.13) is in correspondence with partitions $4=2+2$. In result, the polinomials representing fourth order Casimir of $A_{N}$ Lie algebras are obtained in the following forms:

$$
\begin{aligned}
& y_{1}(N)=-\frac{1}{3} \rho_{4}(N) \\
& y_{2}(N)=\frac{1}{6}(N+1) \rho_{1}(N) \\
& y_{3}(N)=-\frac{1}{6} \rho_{4}(N) \\
& y_{4}(N)=\rho_{2}(N)
\end{aligned}
$$

for subset $T^{(0)}$,

$$
\begin{aligned}
& y_{1}\left(i_{1}, N\right)=\frac{1}{6} \rho_{1}(N)\left(N+1-3 i_{1}\right) \\
& y_{2}\left(i_{1}, N\right)=-\frac{1}{2} \rho_{1}(N) i_{1}
\end{aligned}
$$

for subset $T^{(1)}$,

$$
\begin{aligned}
y_{1}\left(i_{1}, i_{2}, N\right) & =i_{1}\left((N+1) i_{2} \rho_{0}(N)+\rho_{2}(N)\right) \\
y_{2}\left(i_{1}, i_{2}, N\right) & =\frac{1}{2} i_{1}\left(2(N+1) i_{2} \rho_{0}(N)+\rho_{3}(N)\right) \\
y_{3}\left(i_{1}, i_{2}, N\right) & =-(N+1) i_{1}\left(N+1-i_{2}\right) \rho_{0}(N) \\
y_{4}\left(i_{1}, i_{2}, N\right) & =\frac{1}{6}\left(6(N+1) \rho_{0}(N) i_{1} i_{2}\right. \\
& \left.+3 \rho_{3}(N) i_{1}-3 \rho_{1}(N) i_{2}+2(N+1) \rho_{1}(N)\right)
\end{aligned}
$$

for subset $T^{(2)}$,

$$
y\left(i_{1}, i_{2}, i_{3}, i_{4}, N\right)=i_{1}\left(N+1-i_{4}\right)\left(\left(3 i_{2} i_{3}-(N+1)\left(2 i_{2}+i_{3}\right)\right) \rho_{0}(N)+\rho_{1}(N)\right)
$$

for subset $T^{(4)}$.

\section{FIFTH ORDER SOLUTIONS}

In this section we summarize our solutions to equations (II.6) for $\mathrm{p}=5$. As being in line with the former section, we study the whole set of coefficients $g^{A_{1}}, \ldots, A_{5}$ in the following five sub-classes:

$$
\begin{array}{ll}
\left(T^{(0)}\right) & g^{a_{1}, a_{2}, a_{3}, a_{4}, a_{5}}, \\
\left(T^{(1)}\right) & g^{a_{1}, a_{2}, a_{3}, a_{4}, I_{1}}, \\
\left(T^{(2)}\right) & g^{a_{1}, a_{2}, a_{3}, I_{1}, I_{2}}, \\
\left(T^{(3)}\right) & g^{a_{1}, a_{2}, I_{1}, I_{2}, I_{3}}, \\
\left(T^{(5)}\right) & g^{I_{1}, I_{2}, I_{3}, I_{4}, I_{5}} .
\end{array}
$$

There should be no confusion between (IV.1) and (III.1) because the notations are clear. We now want to show that these sub-classes have the following direct sum decompositions in their clusters:

$$
\begin{aligned}
& T^{(0)} \equiv \bigoplus_{k=1}^{4} T^{(0)}(k), \\
& T^{(1)} \equiv \bigoplus_{k=-7}^{13} T^{(1)}(k), \\
& T^{(2)} \equiv \bigoplus_{k=-4}^{6} T^{(2)}(k), \\
& T^{(3)} \equiv \bigoplus_{k=-4}^{6} T^{(3)}(k) .
\end{aligned}
$$


On each particular sub-class, we define the indicators act in the following ways:

$$
\begin{aligned}
& I N D\left(T^{(0)}\right) \equiv \Sigma_{0}\left(\kappa_{1}\left(a_{1}, a_{2}\right), \kappa_{1}\left(a_{1}, a_{3}\right), \kappa_{1}\left(a_{1}, a_{4}\right), \kappa_{1}\left(a_{1}, a_{5}\right), \kappa_{1}\left(a_{2}, a_{3}\right),\right. \\
& \left.\kappa_{1}\left(a_{2}, a_{4}\right), \kappa_{1}\left(a_{2}, a_{5}\right), \kappa_{1}\left(a_{3}, a_{4}\right), \kappa_{1}\left(a_{3}, a_{5}\right), \kappa_{1}\left(a_{4}, a_{5}\right)\right), \\
& \operatorname{IND}\left(T^{(1)}\right) \equiv \Sigma_{1}\left(\Gamma_{11}\left(\kappa_{1}\left(a_{1}, a_{2}\right), \kappa_{1}\left(a_{1}, a_{3}\right), \kappa_{1}\left(a_{1}, a_{4}\right), \kappa_{1}\left(a_{2}, a_{3}\right), \kappa_{1}\left(a_{2}, a_{4}\right), \kappa_{1}\left(a_{3}, a_{4}\right)\right),\right. \\
& \left.\Gamma_{12}\left(\kappa_{2}\left(I_{1}, \alpha_{a_{1}}\right), \kappa_{2}\left(I_{1}, \alpha_{a_{2}}\right), \kappa_{2}\left(I_{1}, \alpha_{a_{3}}\right), \kappa_{2}\left(I_{1}, \alpha_{a_{4}}\right)\right)\right), \\
& \operatorname{IND}\left(T^{(2)}\right) \equiv \Sigma_{2}\left(\Gamma_{2}\left(\kappa_{2}\left(I_{1}, \alpha_{a_{1}}\right), \kappa_{2}\left(I_{1}, \alpha_{a_{2}}\right), \kappa_{2}\left(I_{1}, \alpha_{a_{3}}\right)\right)\right. \\
& \left.\Gamma_{2}\left(\kappa_{2}\left(I_{2}, \alpha_{a_{1}}\right), \kappa_{2}\left(I_{2}, \alpha_{a_{2}}\right), \kappa_{2}\left(I_{2}, \alpha_{a_{3}}\right)\right)\right), \\
& \operatorname{IND}\left(T^{(3)}\right) \equiv \Sigma_{3}\left(\Gamma_{3}\left(\kappa_{2}\left(I_{1}, \alpha_{a_{1}}\right), \kappa_{2}\left(I_{1}, \alpha_{a_{2}}\right)\right),\right. \\
& \Gamma_{3}\left(\kappa_{2}\left(I_{2}, \alpha_{a_{1}}\right), \kappa_{2}\left(I_{2}, \alpha_{a_{2}}\right)\right) \text {, } \\
& \left.\Gamma_{3}\left(\kappa_{2}\left(I_{3}, \alpha_{a_{1}}\right), \kappa_{2}\left(I_{3}, \alpha_{a_{2}}\right)\right)\right) \text {. }
\end{aligned}
$$

It is seen that in all these actions the scalar products are just the same as in the 4th order. This reflects the similarity in the construction of 4 th and 5 th order Casimir operators of $A_{N}$ Lie algebras. As will be explained elsewhere, for 6th and 7th order Casimirs we know that one needs an additional scalar product $\kappa_{3}$ to define corresponding indicators.

Note also that we need to introduce several $\Gamma$-generators in (IV.3). Explicit calculations show that they take the following different values:

$$
\begin{aligned}
& \Gamma_{11}(-2,-2,-2,-2, \quad 2, \quad 2) \equiv \Gamma_{11}(1) \\
& \Gamma_{11}(-1,-1,-1,-1, \quad 0,0) \equiv \Gamma_{11}(2) \\
& \Gamma_{11}(-2,-2,-1,-1, \quad 1,1) \equiv \Gamma_{11}(3) \\
& \Gamma_{11}(-2,-2, \quad 0, \quad 0, \quad 0, \quad 0) \equiv \Gamma_{11}(4)
\end{aligned}
$$

$\Gamma_{12}(1,1,1,1) \equiv \Gamma_{12}(1)$

$\Gamma_{12}(1,1,2,2) \equiv \Gamma_{12}(2)$

$\Gamma_{12}(2,2,2,2) \equiv \Gamma_{12}(3)$

$\Gamma_{12}(-1, \quad 1,2,2) \equiv \Gamma_{12}(4)$

$\Gamma_{12}(-1,-1,1,1) \equiv \Gamma_{12}(5)$

$\Gamma_{12}(-1,-1,-1,-1) \equiv \Gamma_{12}(-1)$

$$
\Gamma_{12}(-1,-1, \quad 2,2) \equiv \Gamma_{12}(-2)
$$

$$
\begin{aligned}
& \Gamma_{2}(1,2,2) \equiv \Gamma_{2}(1), \\
& \Gamma_{2}(1,1,1) \equiv \Gamma_{2}(2) \text {, } \\
& \Gamma_{2}(-1,2,2) \equiv \Gamma_{2}(-1) \text {, } \\
& \Gamma_{2}(-1,-1,-1) \equiv \Gamma_{2}(-2)
\end{aligned}
$$

$$
\begin{aligned}
& \Gamma_{3}(1,1) \equiv \Gamma_{3}(1) \\
& \Gamma_{3}(2,2) \equiv \Gamma_{3}(2) \\
& \Gamma_{3}(-1,-1) \equiv \Gamma_{3}(-1)
\end{aligned}
$$

Both $\Gamma$ and $\Sigma$-generators are assumed to be completely symmetrical in their indices. 
We can continue now in the $A_{5}$ example again. By using above values of $\Gamma$-generators, one can easily show that indicators take the following values on chosen elements of corresponding sub-classes:

$$
\begin{aligned}
& \operatorname{IND}\left(g^{1,1,2,16,21}\right)=\Sigma_{0}(-2,-2,-1,-1,-1,-1,-1, \quad 1, \quad 1,2) \equiv \Sigma_{0}(1) \\
& I N D\left(g^{1,2,3,4,28}\right)=\Sigma_{0}(-1,-1,-1,-1,-1, \quad 0, \quad 0, \quad 0, \quad 0,0) \equiv \Sigma_{0}(2) \\
& I N D\left(g^{1,2,3,16,22}\right)=\Sigma_{0}(-2,-1,-1,-1,-1,-1, \quad 0, \quad 0, \quad 1,1) \equiv \Sigma_{0}(3) \\
& I N D\left(g^{1,2,4,19,21}\right)=\Sigma_{0}(-2,-1,-1,-1, \quad 0, \quad 0, \quad 0, \quad 0, \quad 0,0) \equiv \Sigma_{0}(4) \text {. }
\end{aligned}
$$

$$
\begin{aligned}
& \operatorname{IND}\left(g^{1,1,16,16,32}\right)=\Sigma_{1}(1,1) \equiv \Sigma_{1}(1) \\
& I N D\left(g^{1,2,3,25,34}\right)=\Sigma_{1}(2,1) \equiv \Sigma_{1}(2) \\
& \operatorname{IND}\left(g^{1,2,16,17,33}\right)=\Sigma_{1}(3,1) \equiv \Sigma_{1}(3) \\
& I N D\left(g^{1,3,16,18,34}\right)=\Sigma_{1}(4,1) \equiv \Sigma_{1}(4) \\
& \operatorname{IND}\left(g^{1,2,3,25,33}\right)=\Sigma_{1}(2,2) \equiv \Sigma_{1}(5) \\
& I N D\left(g^{1,2,16,17,32}\right)=\Sigma_{1}(3,2) \equiv \Sigma_{1}(6) \\
& \operatorname{IND}\left(g^{1,3,16,18,33}\right)=\Sigma_{1}(4,2) \equiv \Sigma_{1}(7) \\
& I N D\left(g^{1,1,16,16,31}\right)=\Sigma_{1}(1,3) \equiv \Sigma_{1}(8) \\
& \operatorname{IND}\left(g^{2,10,21,22,32}\right)=\Sigma_{1}(2,3) \equiv \Sigma_{1}(9) \\
& \operatorname{IND}\left(g^{1,6,16,21,31}\right)=\Sigma_{1}(3,3) \equiv \Sigma_{1}(10) \\
& \operatorname{IND}\left(g^{2,10,17,25,32}\right)=\Sigma_{1}(4,3) \equiv \Sigma_{1}(11) \\
& \operatorname{IND}\left(g^{1,2,3,25,32}\right)=\Sigma_{1}(2,4) \equiv \Sigma_{1}(12) \\
& I N D\left(g^{1,3,16,18,32}\right)=\Sigma_{1}(4,5) \equiv \Sigma_{1}(13) \\
& \operatorname{IND}\left(g^{1,2,21,32,32}\right)=\Sigma_{2}(1,1) \equiv \Sigma_{2}(1) \\
& \operatorname{IND}\left(g^{1,2,21,32,33}\right)=\Sigma_{2}(2,1) \equiv \Sigma_{2}(2) \\
& \operatorname{IND}\left(g^{1,2,21,33,33}\right)=\Sigma_{2}(2,2) \equiv \Sigma_{2}(3) \\
& I N D\left(g^{2,3,22,31,33}\right)=\Sigma_{2}(-2,1) \equiv \Sigma_{2}(4) \\
& \operatorname{IND}\left(g^{1,2,21,31,32}\right)=\Sigma_{2}(-1,1) \equiv \Sigma_{2}(5) \\
& \operatorname{IND}\left(g^{2,3,22,31,34}\right)=\Sigma_{2}(-2,2) \equiv \Sigma_{2}(6) \\
& \operatorname{IND}\left(g^{1,16,32,32,32}\right)=\Sigma_{3}(1,1,1) \equiv \Sigma_{3}(1) \\
& I N D\left(g^{2,17,31,32,32}\right)=\Sigma_{3}(-1,2,2) \equiv \Sigma_{3}(2) \\
& \operatorname{IND}\left(g^{2,17,31,33,33}\right)=\Sigma_{3}(-1,1,1) \equiv \Sigma_{3}(3) \\
& I N D\left(g^{1,16,31,32,32}\right)=\Sigma_{3}(1,1,2) \equiv \Sigma_{3}(4) \\
& I N D\left(g^{1,16,31,31,31}\right)=\Sigma_{3}(2,2,2) \equiv \Sigma_{3}(5) \\
& I N D\left(g^{2,17,31,32,33}\right)=\Sigma_{3}(-1,1,2) \equiv \Sigma_{3}(6) \text {. }
\end{aligned}
$$

As in the fourth order calculations, to factor out their rational parts is crucial to obtain polinomial 
expressions for coefficients $g^{A_{1}, A_{2}, A_{3}, A_{4}, A_{5}}$. This is provided by the following appropriate assumptions:

$$
\begin{aligned}
g^{a_{1}, a_{2}, a_{3}, a_{4}, a_{5}} & \equiv g_{5}(N) y_{k}(N), \quad k=1, . .4 \\
g^{a_{1}, a_{2}, a_{3}, a_{4}, I_{1}} & \equiv g_{5}(N) y_{k}\left(i_{1}, N\right), \quad k=1, . .13 \\
g^{a_{1}, a_{2}, a_{3}, I_{1}, I_{2}} & \equiv g_{5}(N) y_{k}\left(i_{1}, i_{2}, N\right), \quad k=1, . .6 \\
g^{a_{1}, a_{2}, I_{1}, I_{2}, I_{3}} & \equiv g_{5}(N) y_{k}\left(i_{1}, i_{2}, i_{3}, N\right) \quad, \quad k=1, . .6 \\
g^{I_{1}, I_{2}, I_{3}, I_{4}, I_{5}} & \equiv g_{5}(N) y\left(i_{1}, i_{2}, i_{3}, i_{4}, i_{5}, N\right)
\end{aligned}
$$

where

$$
g_{5}(N) \equiv \frac{1}{N(N-1)(N-2)(N-3)} .
$$

Note here that there should be no confusion between y-polinomials defined in expressions (III.9) and (IV.10). In all expressions above some $\Sigma$-generators come also together with their conjugates having negative arguments. For instance, $\Sigma_{1}(1)$ has a conjugate $\Sigma_{1}(-1)$ whereas there is no need to introduce a conjugate for $\Sigma_{1}(13)$. This in effect is due to transformations properties of coefficients under duality transformations mentioned in section II. These properties are properly reflected by the following expressions:

$$
\begin{aligned}
& y_{-k}\left(i_{1}, N\right)=(-1)^{1} y_{k}\left(N+1-i_{1}, N\right) \quad, \quad k=1, . .13 \\
& y_{-k}\left(i_{1}, i_{2}, N\right)=(-1)^{2} y_{k}\left(N+1-i_{2}, N+1-i_{1}, N\right) \quad, \quad k=1, . .6 \\
& y_{-k}\left(i_{1}, i_{2}, i_{3}, N\right)=(-1)^{3} y_{k}\left(N+1-i_{3}, N+1-i_{2}, N+1-i_{1}, N\right), k=1, . .6 \\
& y\left(i_{1}, i_{2}, i_{3}, i_{4}, i_{5}, N\right)=(-1)^{5} y\left(N+1-i_{5}, N+1-i_{4}, N+1-i_{3}, N+1-i_{2}, N+1-i_{1}, N\right) .
\end{aligned}
$$

As in (III.12), it would also be useful to define here some auxiliary polinomials $\tau_{r}(N)(r=0, . .8)$ :

$$
\begin{aligned}
& \tau_{0}(N)=-(5 N-9) v(1)+5 N v(2) \\
& \tau_{1}(N)=(N-2)(N-3)((N-7) v(1)-2 N v(2)) \\
& \tau_{3}(N)=\left(N^{3}-2 N^{2}+2 N-3\right) v(1)-N\left(N^{2}+1\right) v(2) \\
& \tau_{2}(N)=(N+1)\left(\left(11 N^{2}-23 N+6\right) v(1)-10 N^{2} v(2)\right) \\
& \tau_{4}(N)=\left(N^{3}+9 N^{2}-21 N+3\right) v(1)-N\left(N^{2}+10 N+1\right) v(2) \\
& \tau_{5}(N)=\left(3 N^{3}+5 N^{2}-17 N-3\right) v(1)-N(N+3)(3 N+1) v(2) \\
& \tau_{6}(N)=-\left(3 N^{3}+16 N^{2}-40 N+3\right) v(1)+N\left(3 N^{2}+20 N+3\right) v(2) \\
& \tau_{7}(N)=\left(9 N^{3}-7 N^{2}-5 N-21\right) v(1)-N\left(9 N^{2}+10 N+9\right) v(2) \\
& \tau_{8}(N)=-\left(6 N^{3}-N^{2}-11 N-12\right) v(1)+2 N\left(3 N^{2}+5 N+3\right) v(2)
\end{aligned}
$$

for which the two free parameters $\mathrm{v}(1)$ and $\mathrm{v}(2)$ are chosen by

$$
y(1,1,1,1,1, N) \equiv v(1) \quad, \quad y(1,1,1,2,2, N) \equiv v(2) .
$$

Note here that the choices (IV.14) are compatible with partitions $5=3+2$. In result, the polinomials assigned to sub-classes $T^{(s)}$ will thus be given as in the following:

$$
\begin{aligned}
& y_{1}(N)=-\frac{1}{12}(N+1) \tau_{1}(N) \\
& y_{2}(N)=\frac{1}{24}(N+1) \tau_{2}(N) \\
& y_{3}(N)=-\frac{1}{24}(N+1) \tau_{1}(N) \\
& y_{4}(N)=-\frac{1}{2}(N+1) \tau_{3}(N)
\end{aligned}
$$

for subset $T^{(0)}$, 


$$
\begin{aligned}
& y_{1}\left(i_{1}, N\right)=\frac{1}{3} i_{1} \tau_{1}(N) \\
& y_{2}\left(i_{1}, N\right)=-\frac{1}{6} i_{1} \tau_{2}(N) \\
& y_{3}\left(i_{1}, N\right)=\frac{1}{6} i_{1} \tau_{1}(N) \\
& y_{4}\left(i_{1}, N\right)=2 i_{1} \tau_{3}(N) \\
& y_{5}\left(i_{1}, N\right)=\frac{1}{24}\left(N+1-4 i_{1}\right) \tau_{2}(N) \\
& y_{6}\left(i_{1}, N\right)=-\frac{1}{24}\left(N+1-4 i_{1}\right) \tau_{1}(N) \\
& y_{7}\left(i_{1}, N\right)=-\frac{1}{2}\left(N+1-4 i_{1}\right) \tau_{3}(N) \\
& y_{8}\left(i_{1}, N\right)=-\frac{1}{6}\left(N+1-2 i_{1}\right) \tau_{1}(N) \\
& y_{9}\left(i_{1}, N\right)=\frac{1}{12}\left(N+1-2 i_{1}\right) \tau_{2}(N) \\
& y_{10}\left(i_{1}, N\right)=-\frac{1}{12}\left(N+1-2 i_{1}\right) \tau_{1}(N) \\
& y_{11}\left(i_{1}, N\right)=-\left(N+1-2 i_{1}\right) \tau_{3}(N) \\
& y_{12}\left(i_{1}, N\right)=\frac{1}{12}\left(N+1-2 i_{1}\right) \tau_{2}(N) \\
& y_{13}\left(i_{1}, N\right)=-\left(N+1-2 i_{1}\right) \tau_{3}(N)
\end{aligned}
$$

for subset $T^{(1)}$,

$$
\begin{aligned}
& y_{1}\left(i_{1}, i_{2}, N\right)=\frac{1}{24}\left(\begin{array}{llll}
12 & i_{1} & i_{2} & \tau_{4}(N)+2\left(N+1-2 i_{2}\right.
\end{array}\right) \tau_{2}(N) \\
& \left.-4 i_{1}(N+1) \tau_{5}(N)\right) \\
& y_{2}\left(i_{1}, i_{2}, N\right)=\frac{1}{12}\left(6 i_{1} i_{2} \tau_{4}(N)-2 i_{1}(N+1) \tau_{5}(N)\right) \\
& y_{3}\left(i_{1}, i_{2}, N\right)=\frac{1}{4}\left(2 i_{1} i_{2} \tau_{4}(N)-2 i_{1}(N+1) \tau_{3}(N)\right. \\
& y_{4}\left(i_{1}, i_{2}, N\right)=\frac{1}{6}\left(N+1-i_{2}\right)\left(\tau_{2}(N)-3 i_{1} \tau_{4}(N)\right) \\
& y_{5}\left(i_{1}, i_{2}, N\right)=\frac{1}{48}\left(24 i_{1} i_{2} \tau_{4}(N)+2\left(3(N+1)-4 i_{2}\right) \tau_{2}(N)\right. \\
& \left.+8 i_{1}(N+1) \tau_{6}(N)\right) \\
& y_{6}\left(i_{1}, i_{2}, N\right)=-\frac{1}{2} i_{1}\left(N+1-i_{2}\right) \tau_{4}(N)
\end{aligned}
$$

for subset $T^{(2)}$, 


$$
\begin{aligned}
y_{1}\left(i_{1}, i_{2}, i_{3}, N\right) & =i_{1} i_{2} i_{3}(N+1) \tau_{0}(N)-i_{1}\left(N+1-4 i_{2}-2 i_{3}\right) \tau_{3}(N) \\
y_{2}\left(i_{1}, i_{2}, i_{3}, N\right) & =\frac{1}{6}\left(N+1-i_{3}\right) \times \\
& \left(3 i_{1}\left(N+1-2 i_{2}\right)(N+1) \tau_{0}(N)+2 \tau_{2}(N)-3 i_{2} \tau_{4}(N)\right) \\
y_{3}\left(i_{1}, i_{2}, i_{3}, N\right) & =-\left(N+1-i_{3}\right)\left(i_{1} i_{2}(N+1) \tau_{0}(N)+2 i_{1} \tau_{3}(N)\right) \\
y_{4}\left(i_{1}, i_{2}, i_{3}, N\right) & =\frac{1}{2}\left(2 i_{1} i_{2} i_{3}(N+1) \tau_{0}(N)\right. \\
& \left.-i_{1}\left(3(N+1)-4 i_{3}\right) \tau_{3}(N)+i_{1} i_{2} \tau_{7}(N)\right) \\
y_{5}\left(i_{1}, i_{2}, i_{3}, N\right) & =-\frac{1}{24} \times\left(12 i_{1} i_{3}\left(N+1-2 i_{2}\right)(N+1) \tau_{0}(N)\right. \\
& -2\left(3(N+1)-4 i_{3}\right) \tau_{2}(N)-12 i_{2} i_{3} \tau_{4}(N) \\
& \left.-4 i_{2}(N+1) \tau_{6}(N)-12 i_{1} i_{2} \tau_{7}(N)-8 i_{1}(N+1) \tau_{8}(N)\right) \\
y_{6}\left(i_{1}, i_{2}, i_{3}, N\right) & =\frac{1}{2} i_{1}\left(N+1-2 i_{2}\right)\left(N+1-i_{3}\right)(N+1) \tau_{0}(N)
\end{aligned}
$$

for subset $T^{(3)}$,

$$
\begin{aligned}
y\left(i_{1}, i_{2}, i_{3}, i_{4}, i_{5}, N\right) & =i_{1}\left(N+1-i_{5}\right) \times( \\
& \left(4 i_{2} i_{3} i_{4}-(N+1)\left(3 i_{2} i_{3}+2 i_{2} i_{4}+i_{3} i_{4}\right)\right) \tau_{0}(N) \\
& \left.+\tau_{2}(N)-i_{4} \tau_{4}(N)-\left(2 i_{2}+i_{3}\right) \tau_{5}(N)\right)
\end{aligned}
$$

for subset $T^{(5)}$.

\section{CONCLUSIONS}

In sections (III) and (IV), our general framework is outlined to construct the most general operatorial forms of fourth and fifth order Casimir elements of $A_{N}$ Lie algebras. The most general here means that everything is expressed in terms of two free parameters which are specified in (III.13) for $\mathrm{p}=4$ and in (IV.14) for $\mathrm{p}=5$. We have obtained some generalizations for $\mathrm{p}=6$ one interesting feature of which is that an additional scalar product $\kappa_{3}$ is also necessary. This procedure could be proceeded step by step beyond sixth order but we do not know at present how many new indicators will be needed as the degree of Casimir operators increases.

The second point which we would like to emphasize here is for the sub-class $T^{(0)}$ of Casimir coefficients $g^{a_{1}, a_{2}, a_{3}, a_{4}}$. As we point out in section (III), it has the form

$$
T^{(0)}=T^{(0)}(1) \oplus T^{(0)}(2) \oplus T^{(0)}(3) \oplus T^{(0)}(4)
$$

in terms of its clusters $T^{(0)}(k), \mathrm{k}=1,2,3,4$. For a given value of rank $\mathrm{N}, T^{(0)}$ contains totally

$$
\operatorname{dim}\left(T^{(0)}\right)=\frac{1}{2}(N+1) N\left(3 N^{2}-5 N+6\right)
$$

number of elements while its clusters have the following dimensions:

$$
\begin{aligned}
& \operatorname{dim}\left(T^{(0)}(1)\right)=1 \operatorname{Binomial}(N+1,2), \\
& \operatorname{dim}\left(T^{(0)}(2)\right)=3 \operatorname{Binomial}(N+1,3), \\
& \operatorname{dim}\left(T^{(0)}(3)\right)=6 \operatorname{Binomial}(N+1,4), \\
& \operatorname{dim}\left(T^{(0)}(4)\right)=3 \operatorname{Binomial}(N+1,4) .
\end{aligned}
$$

Let us note here that these numbers are calculated in view only of invariance property of coefficients $g^{a_{1}, a_{2}, a_{3}, a_{4}}$. 
A similar calculation gives the following results for $g^{a_{1}, a_{2}, a_{3}, a_{4}, a_{5}}$ :

$$
\begin{aligned}
& \operatorname{dim}\left(T^{(0)}(1)\right)=N^{3}-(N+1) \\
& \operatorname{dim}\left(T^{(0)}(2)\right)=\frac{1}{5}\left(N^{5}-5 N^{4}+5 N^{3}+5 N^{2}-6 N+5\right) \\
& \operatorname{dim}\left(T^{(0)}(3)\right)=24 \operatorname{Binomial}(N+1,4) \\
& \operatorname{dim}\left(T^{(0)}(4)\right)=20 \operatorname{Binomial}(N+1,5)
\end{aligned}
$$

with

$$
\operatorname{dim}\left(T^{(0)}\right)=\frac{1}{30}(N+1) N(N-1)\left(11 N^{2}-25 N+36\right)
$$

number of elements totally. For any one of these numbers, there is a coincidence with dimensions of some Weyl orbits of $A_{N}$ Lie algebras. This however could not only be a coincidence because each particular cluster $T^{(0))}(k)$ forms an irreducible sub-space of $T^{(0))}$, that is

$$
\Theta\left(T^{(0))}(k)\right) \equiv T^{(0))}(k) \quad, \quad k=1,2,3,4 .
$$

under Weyl reflections

$$
\Theta\left(g^{a_{1}, a_{2}, a_{3}, a_{4}}\right) \equiv g^{\theta\left(a_{1}\right), \theta\left(a_{2}\right), \theta\left(a_{3}\right), \theta\left(a_{4}\right)} \quad, \quad a_{1}, \ldots a_{4} \in S_{+} \oplus S_{-}
$$

or

$$
\Theta\left(g^{a_{1}, a_{2}, a_{3}, a_{4}, a_{5}}\right) \equiv g^{\theta\left(a_{1}\right), \theta\left(a_{2}\right), \theta\left(a_{3}\right), \theta\left(a_{4}\right), \theta\left(a_{5}\right)} \quad, \quad a_{1}, \ldots a_{5} \in S_{+} \oplus S_{-}
$$

One can therefore said that indicators which are defined in expressions (III.2) and (IV.3) are to be naturally assigned to subsets $T^{(0)}$ if one recals here that the scalar product $\kappa_{1}$ is Weyl invariant. As will be considered in another publication, this leads us to ask some more fundamental symmetry underlying all the picture here.

As a final remark, we point out that Casimir elements of other Lie algebras can be handled in terms of their most appropriate $A_{N}$ sub-algebras. Following examples are $A_{2}, A_{5}, A_{7}, A_{8}$ for respectively $G_{2}, E_{6}, E_{7}, E_{8}$. An exception is seem to be $F_{4}$ which is studied in terms of $B_{4}$ [15] more conveniently.

\section{REFERENCES}

[1] Hermann, R: Lie Groups for Physicists, W.A.Benjamin (1966) N.Y

Carter, R.W: Simple Groups of Lie Type, J.Wiley and sons (1972) N.Y

[2] Biedenharn, L.C: J.Math.Phys. 4, 436-445 (1963)

Perelemov A.M and Popov V.S: Sov.J.Nucl.Phys. 3, 676-680 (1966)

Louck J.D and Biedenharn L.C: J.Math.Phys. 11, 2368-2414 (1970)

Okubo S: J.Math.Phys. 16, 528-535 (1975)

Nwachuku C.O and Rashid M.A: J.Math.Phys. 17, 1611-1616 (1976)

Okubo S: J.Math.Phys. 18, 2382-2394 (1977)

King R.C and Qubanchi A: J.Phys.A, Math.Gen. 11, 1-7 (1978)

Edwards S.A: J.Math.Phys. 19, 164-167 (1978)

Englefield, M.J and King, R.C: J.Phys. A , Math.Gen 13, 2297-2317 (1980)

Berdjis F: J.Math.Phys. 22, 1851-1856 (1981)

Berdjis F and Beslmuller E: J.Math.Phys. 22, 1857-1860 (1981)

Hughes J.W.B and van der Jeugt J:J.Math.Phys. 26, 894-900 (1985)

Bincer A.M and Riesselman K: J.Math.Phys. 34, 5935-5941 (1993)

[3] Okubo S and Patera J: J.Math.Phys.24, 2722-2733 (1983)

Okubo S and Patera J: J.Math.Phys.25, 219-227 (1984)

[4] Scheunert M: J.Math.Phys. 24, 2681-2688 (1983)

Bincer A.M: J.Math.Phys. 24, 2546-2549 (1983) 
[5] Gould M.D et al.: Eigenvalues of Casimir Invariants for Type I Quantum Superalgebras, q-alg/9506019

[6] Kac V.G: Proc.Natl.Acad.Sci. USA 81, 645-647 (1984)

[7] Thierry-Mieg J: Phys. Lett. B156, 199-202 (1985) and Phys. Lett. B171, 163-169 (1986)

[8] Schwarz J.H: The Second String Revolution, hep-th/9607067

[9] Seiberg N. and Witten E. : Electric-Magnetic Duality, Monopole Condensation and Confinement in N=2 Supersymmetric Yang-Mills Theory, hep-th/9407087, Nucl.Phys. B426 , 19 (1994)

Seiberg N. and Witten E : Monopoles, Duality and Chiral Symmetry Breaking in N=2 Supersymmetric QCD, hep-th/9408099, Nucl. Phys. B431, 484 (1994)

[10] Karadayi H.R: J.Math.Phys. 25, 411-417 (1984)

[11] Borel A and Chevalley C: Mem.Am.Math.Soc. 14, 1 (1955)

Chih-Ta Yen: Sur Les Polynomes de Poincare des Groupes de Lie Exceptionnels, Comptes Rendue Acad.Sci. Paris 628-630 (1949)

Chevalley C: The Betti Numbers of the Exceptional Simple Lie Groups, Proceedings of the International Congress of Mathematicians, 2, 21-24 (1952)

Borel A: Ann.Math. 57, 115-207 (1953)

Coleman A.J: Can.J.Math 10, 349-356 (1958)

[12] Racah, G:Rend.Lincei 8, 108-112 (1950)

Racah, G:Lecture Notes reprinted in Ergebnisse Exacta Naturwissenschaften 37, 28-84 (1951)

[13] Gruber, B and O’Raifertaigh, L: J.Math.Phy. 5, 1796-1804 (1964)

[14] Humhreys J.E: Introduction to Lie Algebras and Representation Theory, Springer-Verlag (1972) N.Y.

[15] Bincer A.M: Casimir Operators of the Exceptional Group $F_{4}$ : the chain $B_{4} \subset F_{4} \subset D_{13}$, hep-th9312148 